\title{
Extrusion Pressure Analysis of Powder State Material of Non-Plug Solid Conveying in Feeding Section of Single Screw Extruder
}

\author{
Dali Shi ${ }^{1, a}$, Chunhui Liu ${ }^{1, b}$, Jianhua Qin ${ }^{1, c}$ \\ ${ }^{1}$ School of Mechanical Engineering, Wuhan Institute of Technology, 430073, Wuhan China \\ asdl-002@163.com, ${ }^{\mathrm{b}}$ sdl_002@163.com, ${ }^{\mathrm{C}}$ qinjianhua1964@163.com
}

Keywords: Non-plug flow; Solid conveying; Extrusion pressure; Granular; N-lamina Model.

\begin{abstract}
Using a single screw extruder for extruding pure UHMWPE, one of the necessary conditions is to generate large enough extrusion pressure ${ }^{[4]}$. This article launches a comprehensive and detailed study of raw material of the pure UHMWPE resin powder, establishes a mathematical model of extrusion molding pressure of powder in feeding section, and puts forward the N-lamina model of non-plug solid conveying, which is suitable for the powder state material.
\end{abstract}

\section{Introduction}

Ultra high molecular weight polyethylene (UHMW-PE) is a kind of thermoplastic engineering plastics with linear structure and excellent comprehensive properties. The average molecular weight of UHMW-PE is about 350,000 8,000,000, because of its maximal chain length and very high molecular weight, it has excellent performances which other plastics have nothing comparable to: high resistance to friction, high impact resistance, surface non-sticky and self-lubrication, chemical resistance, and it can also keep excellent mechanical properties in low temperature conditions. It can even replace materials such as carbon steel and stainless steel, etc. Its main application areas include bulk materials handling, food and beverage processing machinery, chemical industry, mining and mineral processing equipment, transport, etc. One of the most widely used application areas is pipeline ${ }^{[9]}$.

The disadvantage of UHMWPE is that its heat resistance (heat distortion temperature) is low, the processing and shaping properties of UHMWPE are poor, which makes it difficult to process UHMWPE in general molding and extrusion equipments. It usually can not use molding methods of ordinary thermoplastic to process UHMW-PE, but with a special process, under high pressure, UHMW-PE particles can be melted and formed into billet or profiles; and then follows subsequent machining if necessary ${ }^{[9]}$.

In order to solve the processing problems of UHMW-PE, a special design must be made on common molding machinery. "Molding in Barrel" is to mold and process the raw material of pure UHMWPE resin powder by a single screw extruder, because the raw material was not modified, after molding and processing, the profile can preserve the maximum excellent performances of raw material of pure UHMWPE ${ }^{[4]}$.

\section{Raw Material of UHMW-PE Resin Powder and Non-Plug Flow Theory}

GUR ${ }^{\circledR}$ UHMW-PE, the raw material of UHMW-PE resin, is a kind of polymer with high viscosity, it is in powder form, its typical particle size ranges from 100 to 200 micrometre ${ }^{[9]}$.

Non-plug flow theory aims at a state in which the material, just entering the screw, has not been compressed into a solid plug, and it takes the "granular" as research object ${ }^{\text {[1-5]. }}$.

Powder Properties and Behaviors of Solid Particles ${ }^{[6]}$

Powder is a collection of various particles sustained by weak forces such as friction force, vander Waals force etc., and is loose body. Position between each particle is easy to change. Powder is an aggregate of independent and loose solid particles, between which there is empty space, which filled with air and water. 
Laminar-flow phenomenon does exist actually. It clearly associates with powder characteristics or behaviors of solid particles, the shear stress of solid particles is proportional to the normal load, and behaviors of solid particles are suitable for the following inequality (1):

$$
\tau \leq f^{\prime} \sigma
$$

Where $f^{\prime}$ is the static friction coefficient between particles, $\tau$ is shear stress, $\sigma$ shows the range of normal force ("pressure").

Granular Computing Model ${ }^{[1-3] ~[6-7]}$

Non-plug flow theory of single-screw extruder emphasizes the "granular" concept, which embodies in the physical model, computation model and constitutive equation etc., which are expressed in the basic theory of soil mechanics. And granular computing model is two below types (2) and (3):

$$
\begin{aligned}
& \sigma_{3}=\xi \sigma_{z} \\
& \sigma_{3}=\frac{\sigma_{x}+\sigma_{y}}{2}-\sqrt{\left(\frac{\sigma_{x}-\sigma_{y}}{2}\right)^{2}+\tau_{x y}^{2}}
\end{aligned}
$$

Where $\xi$ is the coefficient of lateral pressure, $\sigma_{3}$ is the minimum principal stress on the cross section of the material.

To a point in soil, the ratio of lateral compression stress and vertical pressure caused by the lateral stress is called the lateral pressure coefficient $\xi$. That is,

$$
\xi=\frac{\sigma_{x}}{\sigma_{z}}=\frac{\sigma_{y}}{\sigma_{z}}
$$

The ratio of soil lateral swelling strain and vertical compressive strain is called the soil lateral expansion coefficient (or Poisson's ratio), indicated by $\mu$. Soil lateral expansion coefficient is difficult to be measured directly by test methods, and is usually measured based on its relationship with the lateral pressure coefficient.

$$
\begin{aligned}
\sigma_{x} & =\sigma_{y}=\frac{\mu \sigma_{z}}{1-\mu} \\
\xi & =\frac{\mu}{1-\mu}, \text { or } \mu=\frac{\xi}{1+\xi}
\end{aligned}
$$

The following type (8) is established when there is no lateral expansion; as to the situation with the lateral expansion, we can not take $\xi$ for $\frac{\mu}{1-\mu}$. When seeking the dissolution of the constitutive equation of granular, the case is one with lateral expansion.

Constitutive Equation of Granular ${ }^{[1,8]}$

The constitutive equation of granular is established as follows.

$$
\left\{\begin{array}{c}
\sigma_{\mathrm{x}} \\
\sigma_{\mathrm{y}} \\
\tau_{\mathrm{yz}}
\end{array}\right\}=\frac{E}{1-2 \mu \varsigma(1+\mu)-\mu^{2}}\left[\begin{array}{ccc}
1-\mu \varsigma & \mu(1+\varsigma) & 0 \\
\mu(1+\varsigma) & 1-\mu \varsigma & 0 \\
0 & 0 & \frac{1-2 \mu \varsigma(1+\mu)-\mu^{2}}{2(1+\mu)}
\end{array}\right]\left\{\begin{array}{c}
\varepsilon_{\mathrm{x}} \\
\varepsilon_{\mathrm{y}} \\
\gamma_{\mathrm{yz}}
\end{array}\right\}
$$


Extrusion Pressure of Non-plug Solids Conveying in Feeding Section of a Single-screw Extruder

N-lamina Model of Extrusion Pressure of Non-plug Flow Solids Conveying in Feeding Section of a Single-screw Extruder

\section{Physical Model ${ }^{[1-4]}$}

In feeding section, we can analyze the created pressure by the use of non-plug flow theory; according to velocity distribution characteristics of the materials particles, along the groove depth $h$ direction, the material in the spiral groove can be divided into m laminae of material with the same thickness, each lamina is a solid plug.

\section{Mathematical Model ${ }^{[1-4]}$}

\section{Analysis of force and movement of material laminae}

During the material laminae on the section of the screw groove being in a sliding process, they are subject to compressive stress $\sigma$ (specific value of which is $\bar{\sigma}_{2}$ ) on the sliding surfaces between the material lamina and the adjacent upper material, and the adjacent lower material, as well as on the contact surfaces between the material lamina and the screw ribs; they are also subject to friction shear stress $\tau$ in the above four surfaces. The relation of the above two kinds of stress obeys Coulomb friction law.

$$
\begin{aligned}
& \tau=f \sigma \\
& \bar{\sigma}_{2}=\xi \cdot \bar{\sigma}=\xi \cdot \frac{\sigma_{z, i}^{n+1}+\sigma_{z, i}^{n}}{2} \text {, where } \xi \text { is lateral pressure coefficient. }
\end{aligned}
$$

\section{Solution for N-lamina Model of Non-plug Flow Solid Conveying in Feeding Section by Single}

\section{Screw Extruder}

\section{Relationship between Velocity and Stress ${ }^{[1-3]}$}

While having imaginary displacement in -Z direction along Z-axis of screw groove, the materials of any lamina i are affected by several stress, as shown in Fig. 1, and each stress has imaginary displacement $\delta s_{i}$.

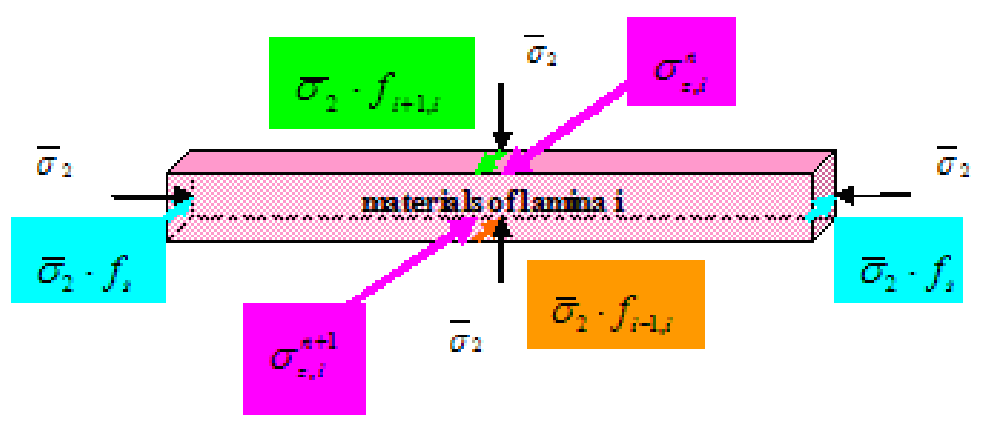

Fig. 1 strength situation of materials of lamina $\mathrm{i}$

By using geometry method of virtual work principle, it is easy to derive the relationship between velocity and stress. List the virtual work equation for the materials of lamina $i$, and then results in equation (9).

$$
\begin{gathered}
\left(K_{a} \cdot \Delta z \cdot w\right) \cdot \bar{\sigma}_{2} \cdot f_{i+1, i} \cdot\left(v_{i+1}-v_{i}\right)+\left(K_{a} \cdot \Delta z \cdot w\right) \cdot \bar{\sigma}_{2} \cdot f_{i-1, i} \cdot\left(v_{i-1}-v_{i}\right)-2\left(K_{a} \cdot \Delta z \cdot \frac{h}{m}\right) \cdot \bar{\sigma}_{2} \cdot f_{s} \cdot v_{i} \\
=\left(K_{z} \cdot \frac{h}{m} \cdot w\right) \cdot\left(\sigma_{z, i}^{n+1}-\sigma_{z, i}^{n}\right) \cdot v_{i}
\end{gathered}
$$

In the above equation: $\Delta z$-longitudinal discrete length of screw groove, or thickness of materials of lamina i in $\mathrm{z}$ direction; $\mathrm{w}$ - width of screw groove; $z$ — flow channel length in feeding section 
which belongs to non-plug flow solid conveying; $\mathrm{m}$ - the total number of the laminae of materials, into which materials in screw groove is separated; $\mathrm{h}$ - depth of screw groove; $K_{a}$-modification coefficient in contact area; $K_{z}$-modification coefficient of axial pressure area.

$k$ is defined by the formula (10), which represents the growth trend of compressive stress.

$$
k=\frac{\left(K_{z} \cdot \frac{h}{m}\right)\left(\sigma_{z}^{n+1}-\sigma_{z}^{n}\right)}{\left(K_{a} \cdot \Delta z\right) \cdot \bar{\sigma}_{2}}
$$

\section{Average Velocity ${ }^{[3]}$}

For a defined number of lamina, namely $m$, the average velocity of all discussed successive laminae can be obtained.

$$
v=\frac{B_{3}}{m} \cdot \frac{f_{4}\left(A_{1}, A_{2}, B_{1}\right) \cdot B_{2}}{f_{3}\left(A_{1}, A_{2}, A_{3}, B_{1}\right)}
$$

\section{Equation of m-th Power of Coefficient $k^{[1-3]}$}

In order to get the value of coefficient $k$, three parameters, $\mathrm{Q}, \psi, V$, are defined as following:

$\mathrm{Q}$-is the output of a single-screw extruder; $\psi$-is a coefficient representing the reciprocal of the output Q which is nondimensional, and satisfies equations $\psi=\frac{B_{3} h w}{Q}$ and $Q=V h w ; V$ - is the average velocity of the successive laminae.

Then equation (12), which is a equation of m-th power of coefficient $k$, can be constructed, and the required value of coefficient $k$ can be gotten when solves equation (12).

$$
k^{m}+a_{1} \cdot k^{m-1}+a_{2} \cdot k^{m-2}+\cdots+a_{m-1} \cdot k+a_{m}=0
$$

\section{Integral Equation and Extrusion pressure ${ }^{[3]}$}

By the definition of parameter $k$, equation (10), the relationship of $\sigma_{z, i}^{n+1}$ and $\sigma_{z, i}^{n}$, compressive stress on front end and rear end of the materials of lamina $i$, can be obtained as equation (13).

$$
\begin{aligned}
& s_{0} \cdot \Delta z=\frac{\Delta \sigma_{z}^{n}}{2 \cdot \sigma_{z}^{n}+\Delta \sigma_{z}^{n}} \\
& \text { Where } s_{0}=k \cdot \frac{K_{a}}{K_{z}} \cdot \frac{m \cdot \xi}{2 h}, \Delta \sigma_{z}^{n}=\sigma_{z}^{n+1}-\sigma_{z}^{n} .
\end{aligned}
$$

Then equation (14) can be inferred by simplification, integration, and stress boundary condition (when $\mathrm{Z}=0, \sigma_{z}=p_{0}$ ). Where $p_{0}$-initial pressure in feeding section of the screw, its normal value is $0.1 \mathrm{MPa} ; p_{1}$-pressure where flow channel length is $\mathrm{z}$ in feeding section of the screw.

$$
p_{1}=p_{0} \cdot \exp \left(k \cdot \frac{K_{a}}{K_{z}} \cdot \frac{m \cdot \xi}{h} \cdot z\right)
$$

The extrusion pressure formula of "N-lamina model" can basically reflect the change tendency of the extrusion pressure. The relationship between three kinds of friction coefficient and $k$ is shown in Fig. ${ }^{[8]}$. 


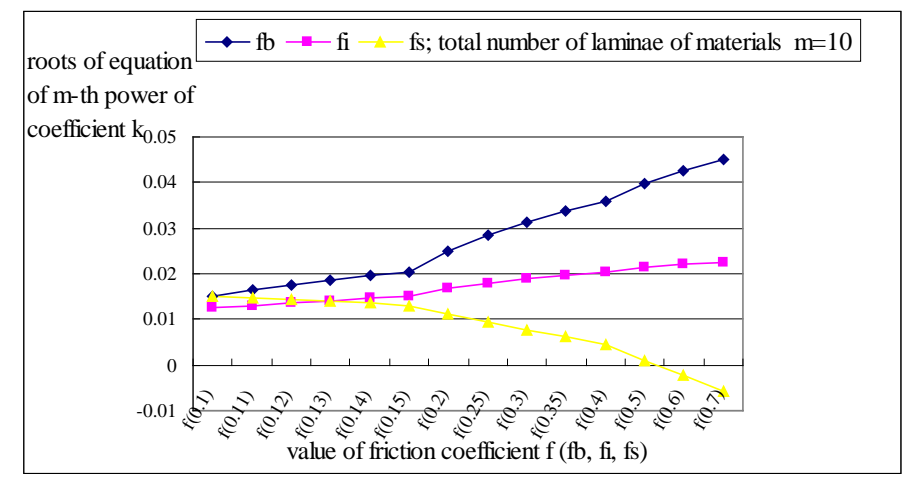

Fig.2 relationship between three kinds of friction coefficient and $k$

\section{Summary}

1) "N-lamina model of non-plug flow solid conveying in feeding section by Single Screw Extruder" put forward by our research group can be applied to the powder state or grain state materials which have laminar flow characteristics, and are being in the feeding section. Then materials can be divided into multi-lamina or any number of laminae when solving extrusion pressure.

2) Pressurization process and trend of the materials in feeding section expressed by the extrusion pressure formula of "N-lamina model" is consistent with the actual situation.

\section{References}

[1] Fuhua Zhu: Extrusion Theory and Application (China Light Industry Publishing House, Beijing 2001).

[2] Shunliang Jiang, Fuhua Zhu: Trilamina Method of Non-plug Solid Conveying in Single-screw Extruder (China Plastics Industry, Sichuan 1997, 2; 83-88).

[3] Chunhui Liu, Jianhua Qin, Dali Shi: N-lamina Model of Extrusion Pressure of Non-Plug Flow Solid Conveying in Feeding Section by Single-screw Extrusion Molding, Advanced Materials

Research (2010).

[4] Jianhua Qin, Guangjun Wang: The research on UHMWPE single screw extruding process theory (Materials for Mechanical Engineering Feb 2007: 34 37).

[5] LunYang, ShuxianXie translated: Experiment Manual of Powder Engineering (written by Sanshumaoxiong, Rigaozhongzhu 1984 Japan).

[6] Zehev Tadmor Costas G.Gogos: Principles of Polymer Processing (A Wiley-Interscience Publication, American 1979).

[7] FanZhu, DaiwenHu: Soil Mechanics (Chongqing University Press, Chongqing 2005)

[8] Chunhui Liu, Dali Shi, Yanyang Wu, Jianhua Qin: Numerical Simulation of Extrusion Pressure of Non-plug Flow Solid Conveying in Feeding Section (Advanced Materials Research Vols. 250-253 (2011) pp 4046-4051).

[9] Guangjian Liu: Ultra high molecular weight polyethylene (UHMW-PE) (Chemical industry publishing house, Beijing, 2001) 